\title{
Self-organised dynamic recognition states for chaotic neural networks
}

\author{
Nigel Crook *, Tjeerd olde Scheper, Vasantha Pathirana \\ Pattern Recognition Research Group, School of Computing and Mathematical Sciences, Oxford \\ Brookes University, Wheatley Campus, Wheatley, Oxford OX33 $1 \mathrm{HX}, \mathrm{UK}$
}

Received 5 August 2000; accepted 7 April 2001

\begin{abstract}
Chaos offers several advantages to the Engineer over other non-chaotic dynamics. One is that chaotic systems are often significantly easier to control than other linear or non-linear systems, requiring only small, appropriately timed perturbations to constrain them within specific unstable periodic orbits (UPOs). Another is that chaotic attractors contain an infinite number of these UPOs. If individual UPOs can be made to represent specific internal states of a system, then a chaotic attractor can be turned into an infinite state machine. In this paper we investigate this possibility with respect to chaotic neural networks. We present a method by which a network can self-select UPOs in response to specific input values. These UPOs correspond to network recognition states for these input values.

(c) 2002 Elsevier Science Inc. All rights reserved.
\end{abstract}

Keywords: Control of chaos; Chaotic neural networks; Adaptive delays

\section{Introduction}

This research is inspired by the discovery that the firing behaviour in biological neural networks is fundamentally chaotic [3-6,10]. Although there is little doubt about the presence of chaos in such networks, there is far less

\footnotetext{
${ }^{*}$ Corresponding author. Tel.: +44-1865-484526/483671; fax: +44-1865-484545/483666.

E-mail address: ntcrook@brookes.ac.uk (N. Crook).
} 
certainty about its role. Some have suggested that biological networks use this chaotic behaviour as a means of storing and retrieving memories [4,9,15-17]. These memories correspond to dynamic "states" which are embedded within the chaotic attractor followed by the network. The use of chaos to store and retrieve memories in an artificial neural network offers a number of significant advantages over alternative models. Among them are a dramatically increased capacity for memory storage, and an ability to "control" the network using only small perturbations [1].

A chaotic system can be described by a set of dynamic variables. An $n$-dimensional plot of an $n$ variable system is called a "state space". Each point in the state space corresponds to a unique set of values for the dynamic variables. As the system changes states a continuous trajectory is described through the state space. Chaotic systems that can be modelled by a set of continuous dynamic variables are constrained to a specific sub-area of state space called an "attractor". An example of an attractor is given in Fig. 1. This attractor is followed by Rössler system of equations [12] which is modelled by three dynamic variables.

By definition, a chaotic system can never be in the same state twice on a continuous run. In other words, a single trajectory in state space can never intersect itself. However, as the system follows its attractor it will come very close to points in state space which it had previously visited. Such trajectories are called unstable periodic orbits (UPOs). A chaotic system following a UPO

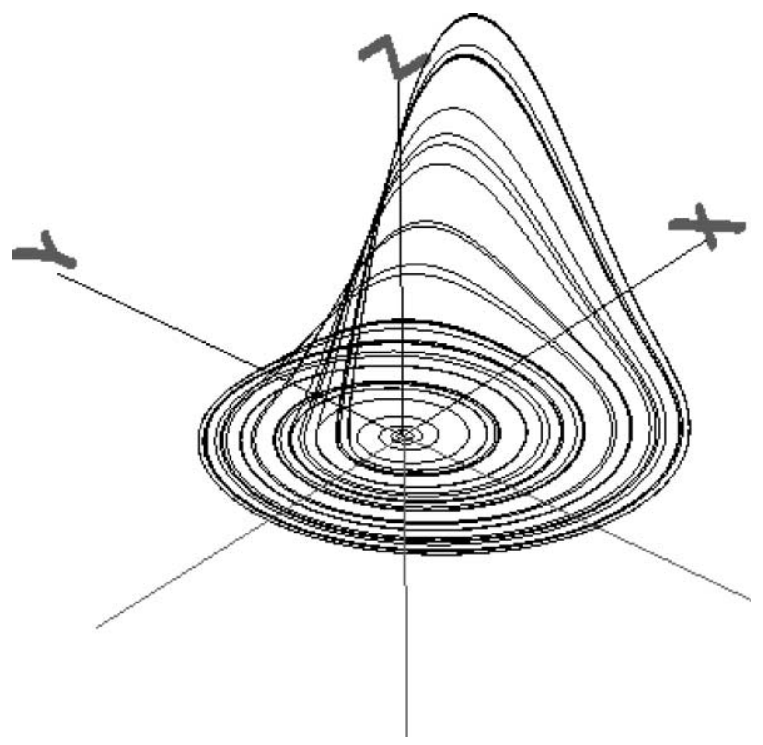

Fig. 1. The attractor for the Rössler system. 


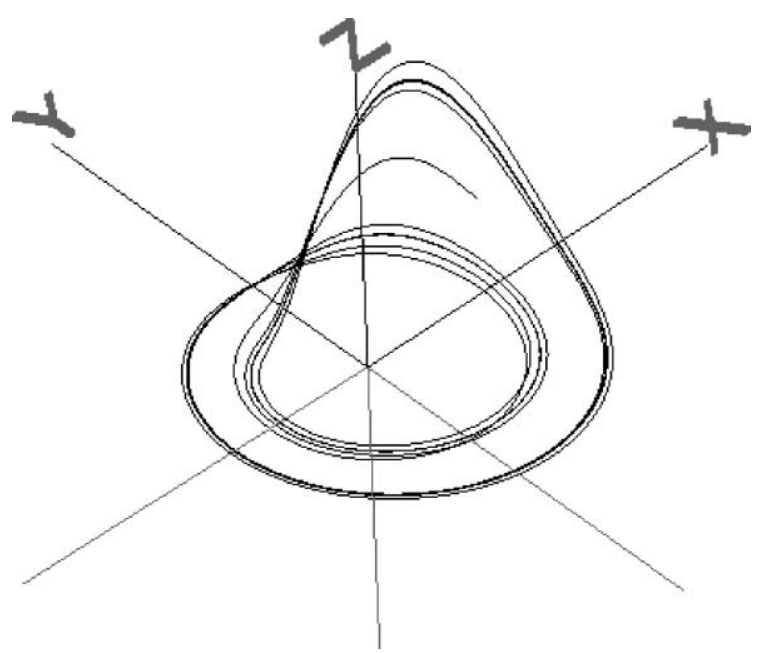

Fig. 2. An unstable periodic orbit embedded in the Rössler attractor.

would describe an orbit in state space which almost repeats itself. However, under the influence of chaos, the trajectory would drift away from this almost periodic orbit and continue to track the surface of the attractor. An example of a UPO is illustrated in Fig. 2.

Techniques have been developed in non-linear dynamical systems theory for controlling a chaotic system so that it will remain on a periodic orbit until the control is removed $[2,5,11,13,14]$. These techniques can be divided into two categories: those that rely on some knowledge of the local dynamics of the attractor around the UPO to be stabilised, and those which use delayed feedback as a method of control. With the first category, the control waits for the system state to fall in the neighbourhood of the desired UPO. Once this has happened, the control makes small temporary adjustments to local dynamics of the attractor so that the system trajectory will come back to the same point when it returns to that neighbourhood, thereby stabilising the orbit. The second category of control method uses a feedback mechanism with a delay proportional to the period of the orbit to be stabilised. The control makes small temporary modifications to the dynamics of the system so that the trajectory will return close to a point in state space which it previously visited. This point is determined by the delay.

Within the context of chaotic neural networks, delayed feedback is the most appropriate method of control. There are three main reasons for this that are based on the biological plausibility of the method in question. The first is that the delay feedback method does not rely on a priori knowledge of the local dynamics of the attractor around the UPO to be stabilised. It seems extremely unlikely that biological neural networks are aware of the local dynamics in 
every UPO. The second is that the delayed feedback method does not specify which UPO is to be stabilised, it simply specifies the period of the required orbit. This suggests an element of self-organisation which is more biologically appealing than the first method. The third reason is that delays are inherent in biological neural networks: the nerve impulse takes time to travel the length of the axon to its target neurons, these neurons, in turn, take time to summate their inputs and produce their response. Consequently, we consider the delayed feedback method to be best suited to the control of chaos in neural networks.

Our approach has been to study small networks consisting of two or three interconnected neurons. We use systems of chaotic equations to model the activation functions of the neurons. Our method for the control of chaos is based on that presented by Kittel et al. [8] and Pyragas [11].

\section{Controlling chaos using delays}

Pyragas' delayed feedback method of controlling chaos assumes that we have a continuous time system which has an output variable, say $y(t)$, that can be measured, and an input signal, $F(t)$ :

$$
\begin{aligned}
& \frac{\mathrm{d} y}{\mathrm{~d} t}=P(y, \boldsymbol{x})+F(t) \\
& \frac{\mathrm{d} \boldsymbol{x}}{\mathrm{d} t}=\boldsymbol{Q}(y, \boldsymbol{x})
\end{aligned}
$$

Here, $P(y, \boldsymbol{x})$ and $\mathbf{Q}(y, \boldsymbol{x})$, which govern the chaotic dynamics of the system, and $\boldsymbol{x}$, which denotes all of the remaining system variables, are assumed to be unknown. When the control signal $F(t)$ is zero, the system (1) is governed by a chaotic attractor.

The input signal $F(t)$ is proportional to the difference between the value of $y$ at time $t$ and the value of $y$ at time $t-\tau$, where $\tau$ is a fixed delay:

$$
F(t)=k[y(t)-y(t-\tau)]
$$

Input signal $F(t)$ attempts to nudge the system back to a state in which output variable $y$ repeats the same value it had at the earlier time specified by the delay $\tau$. In this way, $F(t)$ encourages the system to follow a periodic trajectory with periodicity $\tau$ (Fig. 3).

As the system approaches the periodic trajectory, $F(t)$ will become very small. Fig. 4 shows a time series for the Rössler system. The controlling input signal is initially zero and the system follows its chaotic attractor for a period of time. When the input signal is activated the system quickly converges to a period one UPO. Fig. 4 shows a burst of activity in $F(t)$ which the system is brought under control. Subsequently, as the system moves into the UPO, $F(t)$ becomes very small. 


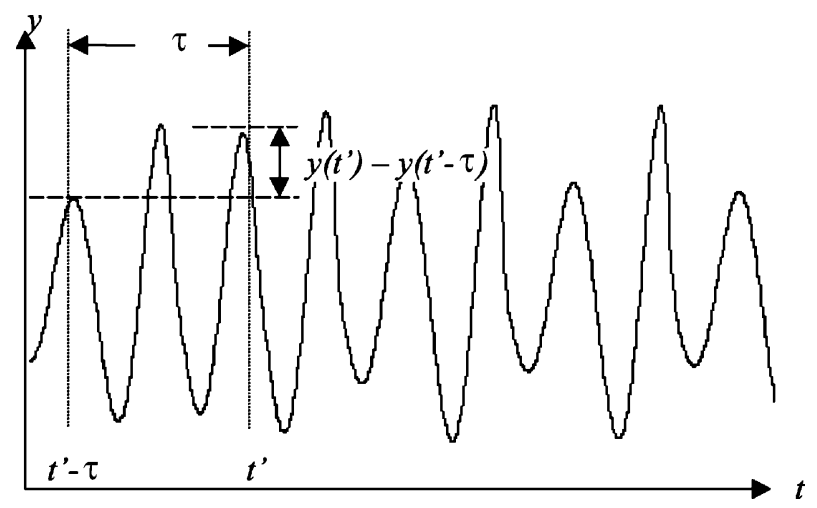

Fig. 3. A sample time series in $y$ with the time delay $\tau$ superimposed.

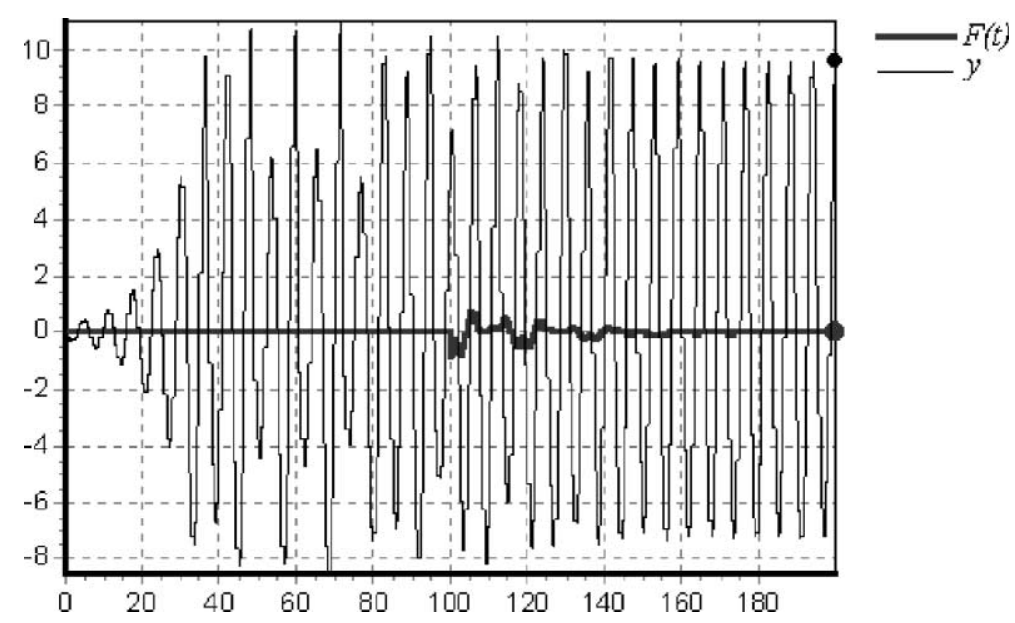

Fig. 4. Time series plot for one of the Rössler equation variables, superimposed on a plot of $F(t)$.

UPOs of varying periods and periodicities can be controlled by this method. Some UPOs are very unstable and require a greater amount of control than others, resulting in an input signal which does not tend towards zero. Furthermore, this control method is sensitive to the choice of $\tau$ and $k$ : UPOs cannot be stabilised for several sub-ranges of values of these control variables.

We have adapted this method of delayed feedback control so that the time delay $\tau$ is not constant, but is a variable of the system whose value is determined in a self-organised manner. Before describing in detail this method of dynamically adapting the delay time, we will consider the Biological justification for adopting such an approach. 


\section{Biological rationale for adaptive delays}

In biological neuronal systems, the action potentials generated by the soma of a neuron travel the length of the axon and are transmitted to other neurons via synaptic connections. In most cases these synapses are located on the dendritic trees of the receiving neurons. Impulses from synapses will cause a sharp rise in the intercellular voltage of the receiving neuron which spreads along the dendritic tree towards the soma. If the voltage rise is sufficiently high at the soma as a result of integrating all inputs on the dendrite, an action potential is generated to travel down the axon of this neuron. Therefore, whenever an action potential is generated, it takes time for the impulse to travel to the soma of the receiving neurons. Fig. 5 illustrates this schematically.

Typically, a neuron will have many synaptic connections with the dendritic tree of a receiving neuron. Since each of these connections will be located at a different point on the dendrite, and so each will result in a different time delay for the signals between the sending and receiving neuron (Fig. 6). According to Hebb [7], those synapses which result in an action potential in the receiving

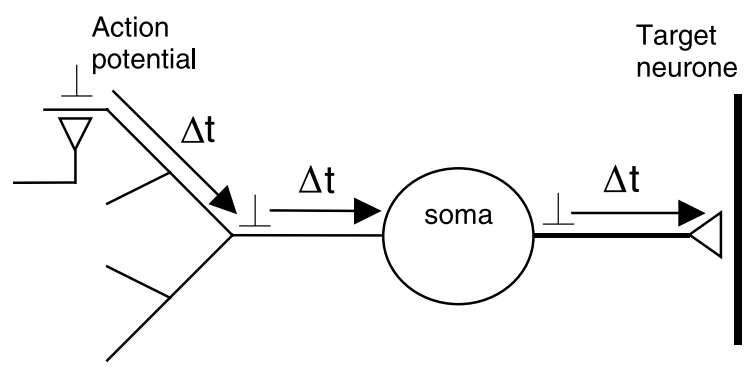

Fig. 5. A schematic diagram of the delay between the initiation of an action potential, and its arrival at the target neuron.

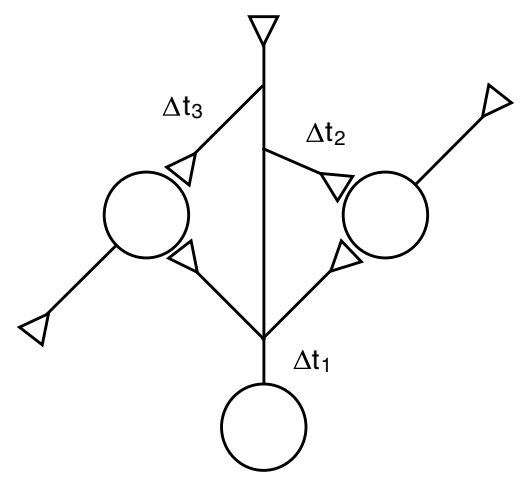

Fig. 6. A schematic diagram of multiple delayed connections between neurons. 
neuron are strengthened, those which do not are weakened. A single impulse from a synapse is rarely sufficient to cause a cell to fire. It is more often the case that several coincident impulses from synapses are needed to provoke a response. This means that those synapses which result in impulses that are appropriately timed will be strengthened. In other words, the adaptive process involves not only the selection of synapses, but also the selection of impulse delays. The method of chaos control we present below is based on this principle of adaptation of delays.

\section{Adaptive delays}

We have modified Pyragas' method of chaos control so that the length of the feedback delay $\tau$ is a variable of the system. We have made $\tau$ proportional to the amount of effort needed to control the system in its current orbit. If the amount of control needed is small, then no change is required in $\tau$, since an orbit with a suitable delay has been successfully stabilised. However, if the system is struggling to control an orbit, then $\tau$ needs to be modified so that the length feedback delay is commensurate with an existing UPO of the system. The equation for adapting $\tau$ is as follows:

$$
\frac{\mathrm{d} \tau}{\mathrm{d} t}=\alpha F \frac{\mathrm{d} y}{\mathrm{~d} t}
$$

where $\alpha$ is a constant. Eq. (3) will be minimised when $F$ becomes small and $\mathrm{d} y / \mathrm{d} t$ is periodic. We have applied this method to the Rössler system of equations [12]. We have modelled these equations using a small network of neurons (Fig. 7) whose activations are defined as follows:

$$
\begin{aligned}
& \frac{\mathrm{d} x_{1}}{\mathrm{~d} t}=w_{12} x_{2}+w_{13} x_{3} \\
& \frac{\mathrm{d} x_{2}}{\mathrm{~d} t}=w_{21} x_{1}+w_{22} x_{2}+F(t, \tau)
\end{aligned}
$$

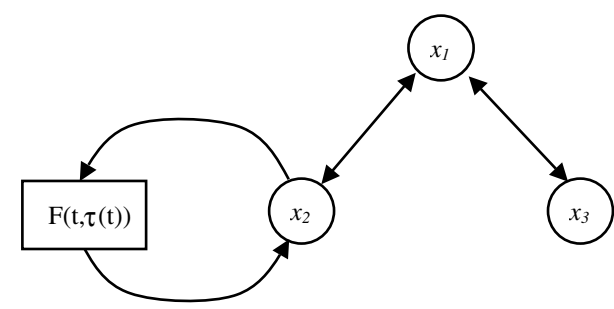

Fig. 7. A three unit model with delayed feedback and external input. 


$$
\frac{\mathrm{d} x_{3}}{\mathrm{~d} t}=v+x_{3} x_{1}+w_{33} x_{3}
$$

where $w_{i j}$ is the weight to unit $i$ from unit $j$. The weights prior to adaptation in this system are given in Table 1 and correspond to the constants used in the Rössler system.

The control of this systems is achieved through the rightmost term in Eq. (5), as defined by Eqs. (2) and (3).

In this paper we present the results of a set of experiments which investigate the dynamics of this system for a range of initial values of $\tau$. The purpose of these experiments was to see if Eq. (3) would be able to adapt $\tau$ towards a nearby controllable orbit. In each experiment, the model was allowed to follow its chaotic attractor until $t=100$, at which point the control was activated using one of the initial values of $\tau$. The results of these experiments are given in Table 2. The initial values for $\tau$ range from 4 to 20 , increasing in steps of 2 . In 6 out of 9 of these initial values the system managed to adapt $\tau$ to a delay value which corresponded to a UPO. Apart from experiment 4 in which $\tau$ went negative, the final values of $\tau$ for these stabilised UPOs were $\sim 5.83$ for a period

Table 1

Weights for the Rössler system

\begin{tabular}{lc}
\hline Weight & Value \\
\hline$w_{12}$ & -1 \\
$w_{13}$ & -1 \\
$w_{21}$ & 1 \\
$w_{22}$ & 0.2 \\
$w_{33}$ & -5.7 \\
$v$ & 0.2 \\
\hline
\end{tabular}

Table 2

Results for Experiment 1

\begin{tabular}{lrrlll}
\hline $\begin{array}{l}\text { Experiment } \\
\text { no. }\end{array}$ & Initial $\tau$ & Final $\tau$ & Max $F$ & Min $F$ & UPO period \\
\hline 1 & 4 & 5.83 & 0.01 & -0.01 & 1 \\
2 & 6 & 17.46 & 1.0 & -1.1 & - \\
3 & 8 & 5.84 & 0.01 & -0.01 & 1 \\
4 & 10 & -25.60 & 0.9 & -0.9 & 1 \\
5 & 12 & 11.66 & 0.01 & -0.01 & 2 \\
6 & 14 & 11.76 & 0.03 & -0.03 & 2 \\
7 & 16 & 17.54 & 0.8 & -0.8 & - \\
8 & 18 & 23.53 & 0.8 & -0.8 & - \\
9 & 20 & 11.72 & 0.06 & -0.04 & 2 \\
\hline
\end{tabular}


1 UPO and $\sim 11.72$ for a period 2 UPO. These values are in close agreement with the results obtained by Pyragas [11] which were 5.9 for a period 1 UPO and 11.75 for a period 2 UPO.

Table 2 shows the maximum and minimum values of $F(t, \tau)$ when a UPO was stabilised. Small absolute values of $\operatorname{Max} F$ and Min $F$ indicate that very little effort was required to keep the system in that UPO. Note that for those experiments which did not result in a stabilised orbit (experiments 2, 7 and 8) $F(t, \tau)$ fluctuates over a relatively wide range of values, indicating that the system is struggling to gain control of the orbit. Figs. 8, 10 and 12 show time series plots of $F(t, \tau)$ and $\tau$ for experiments 1,5 and 7 respectively. Figs. 9, 11 and 13 show the state space in the $x_{1}$ versus $x_{2}$ plane.

Fig. 8 shows that $\tau$ immediately jumps from 4 to a value close to 5.9 as soon as the control is activated at $t=100$. This is in response to large fluctuations in $F(t, \tau)$. Once $\tau$ is close to the periodicity of the UPO at 5.9, only small adjustments are made to its value until it reaches a steady state at 5.83 where the period 1 orbit has been stabilised (Fig. 9). Fig. 10 shows $\tau$ starting at a value of 12 and dropping steadily to the nearby UPO at 11.66. $F(t, \tau)$ does not need to exert much control over the system to maintain this period 2 orbit (Fig. 11). Fig. 12 shows the time series for $F(t, \tau)$ and $\tau$ for experiment 7 , where the delayed feedback was not able to bring the system under control for any orbit (Fig. 13), even on an extended run up to $t=800$. As this graph shows, $\tau$ does not show any signs of approaching a steady state.

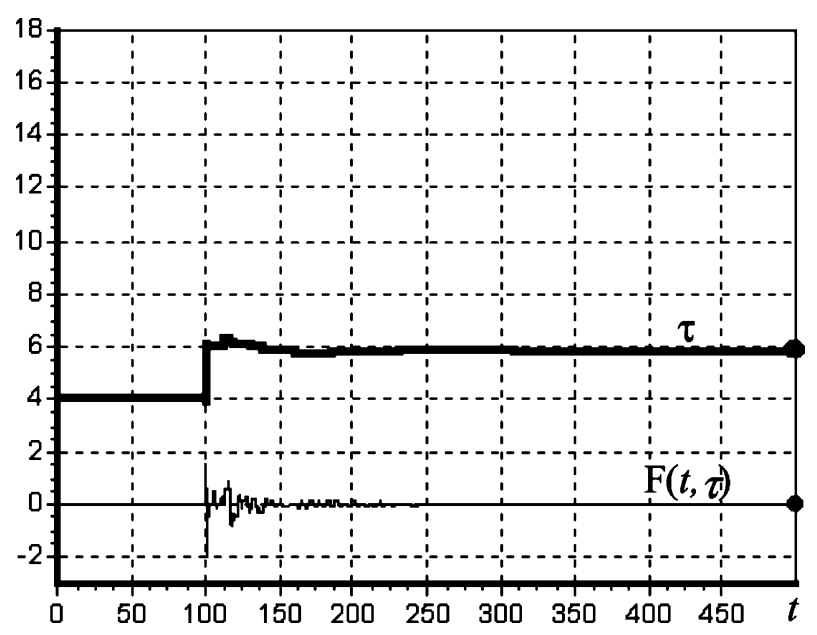

Fig. 8. $F(t, \tau)$ and $\tau$ for experiment 1 . 


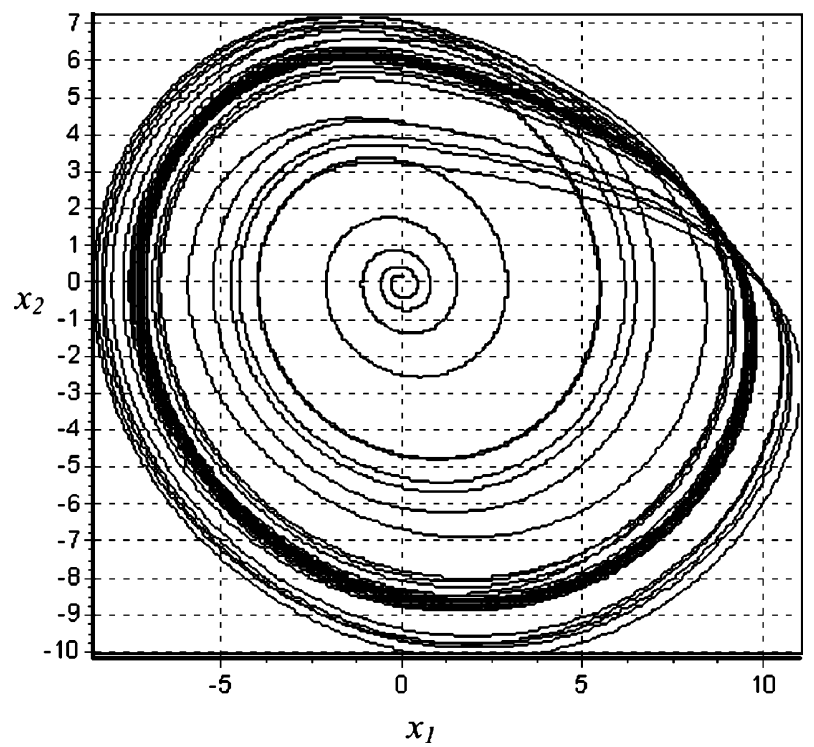

Fig. 9. State space for experiment 1.

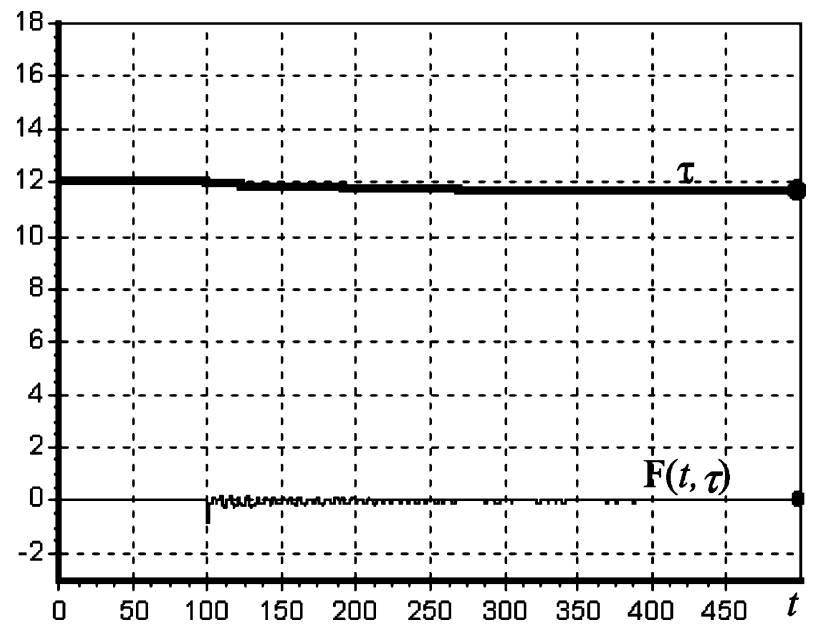

Fig. 10. $F(t, \tau)$ and $\tau$ for experiment 5 .

\section{Stabilizing orbits by frequency modulated input}

A basic premise of our research is that a chaotic neural network will be able to self-select a UPO in response to specific input patterns. Furthermore, each 


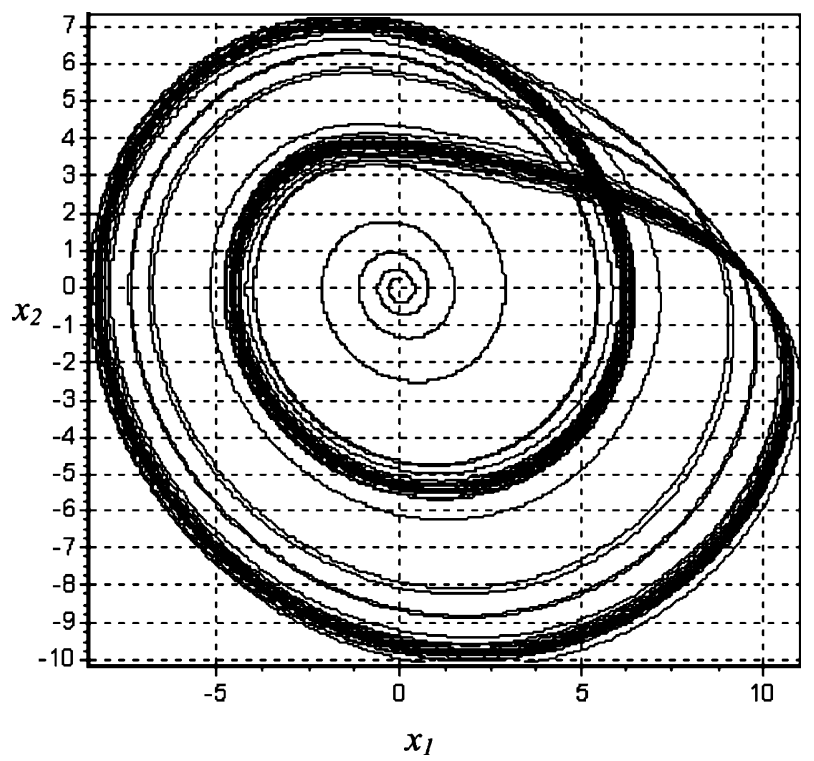

Fig. 11. State space for experiment 5.

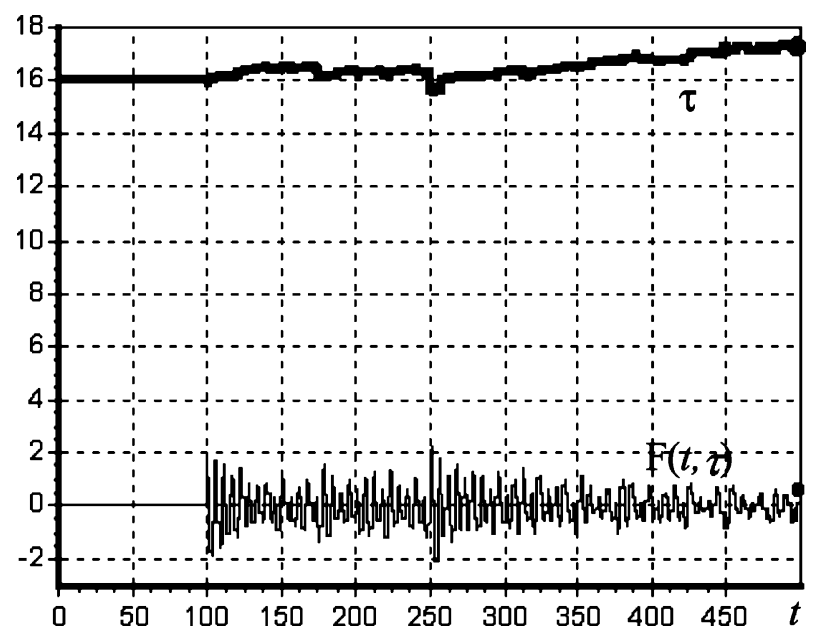

Fig. 12. $F(t, \tau)$ and $\tau$ for experiment 7 .

orbit selected must be unique to that class of input. In this section we demonstrate that this is possible using a periodic square wave input signal to a chaotic network which is under feedback control. In these experiments, $\tau$ was held constant and the periodic input signal was added to $x_{1}$ : 


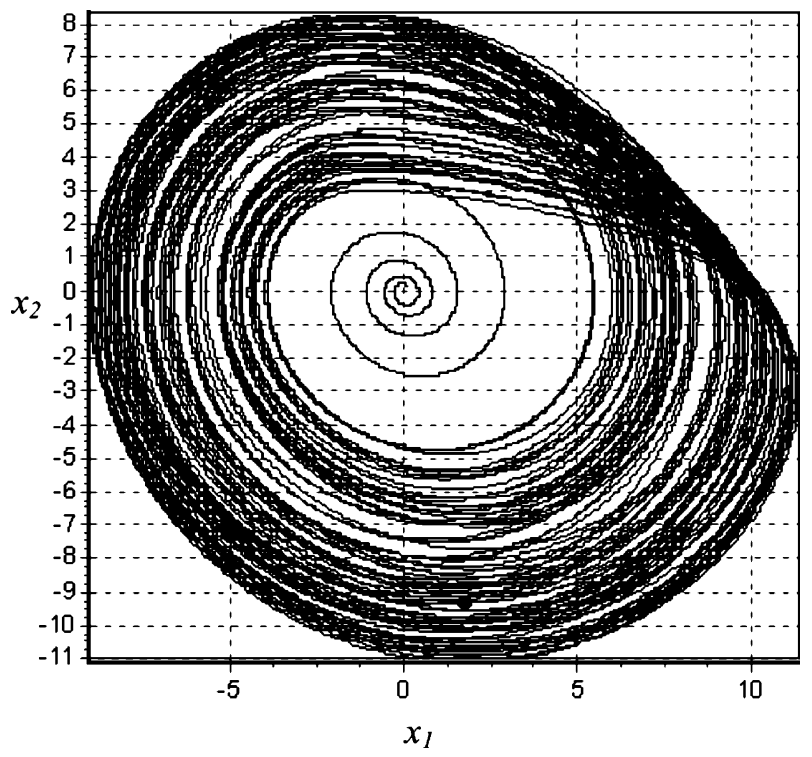

Fig. 13. State space for experiment 7.

$$
\begin{aligned}
& \frac{\mathrm{d} x_{1}}{\mathrm{~d} t}=w_{12} x_{2}+w_{13} x_{3}+I(t) \\
& \frac{\mathrm{d} x_{2}}{\mathrm{~d} t}=w_{21} x_{1}+w_{22} x_{2}+F(t) \\
& \frac{\mathrm{d} x_{3}}{\mathrm{~d} t}=v+x_{3} x_{1}+w_{33} x_{3}
\end{aligned}
$$

All other weights and constants are as described in Table 1. Delayed feedback control is activated simultaneously with the presentation of an external stimulus.

Input values are represented by frequency modulations of the pulse signal $I(t)$, with distinct frequencies (and their sub-harmonics) representing distinct input values. Chaotic systems are sensitive to variations in both frequency and amplitude. However, with large amplitudes it is possible to override the natural dynamics of the network's chaotic attractor. Furthermore, the electrochemical axonal output signals from biological neurons have constant amplitude, with information largely being conveyed in the frequency modulations of the pulse trains. For these reasons we have chosen to use a constant amplitude, frequency modulated input signal.

In the experiments presented here an input pulse amplitude of 1.0 and a pulse width of 3.0 was used. In each experiment the network model was evaluated for a number of time steps prior to the presentation of external input 
to allow initial transients in the network dynamics to decay as the attractor was approached. The delayed feedback control $F(t)$ was then activated simultaneously with the external input $I(t)$. The delay period $\tau$ was held constant for each experiment. The peak values of $x_{1}$ were recorded for pulse periods of $I(t)$ in the range 4-20 (the pulse period is the time between pulses plus the pulse width). The experiments were repeated for delays $\tau_{1}=5.8, \tau_{2}=11.4$ and $\tau_{3}=17.0$ which correspond to the period one, two and three UPOs (respectively) that can be stabilised when delayed feedback is activated. Fig. 14 shows plots of peak $x_{1}$ values versus the period of the pulse for each of the three values of $\tau$.

The plots in Fig. 14 demonstrate that UPOs can only be stabilised for frequencies of the input signal which are simple harmonics of the delay period. In other words, particular delay periods will select corresponding input frequencies and their sub-harmonics. This is confirmed by Fig. 15 which shows plots of $x_{1}$ versus $x_{2}$ for four different periods of the pulse once the transients have died away. Here we make the feedback delay $\tau=5.84$. Plots 1,2 and 3 are stable period 1, 2 and 3 orbits for pulse periods equal to 5.84, 11.64 and 17.52 respectively. These pulse periods are integer multiples of the feedback delay and so illicit a periodic response from the network. Plot 4 shows that a UPO could not be stabilised by a pulse input with period 14.0, which is not an integer multiple of the feedback delay.

In a separate experiment we investigated the affects of activating feedback control before presenting the external input. Fig. 16 below shows the time series of the $x_{1}$ variable for this experiment. The chaotic dynamics were allowed to evolve without any feedback control or external input for $t=0$ to 250 .
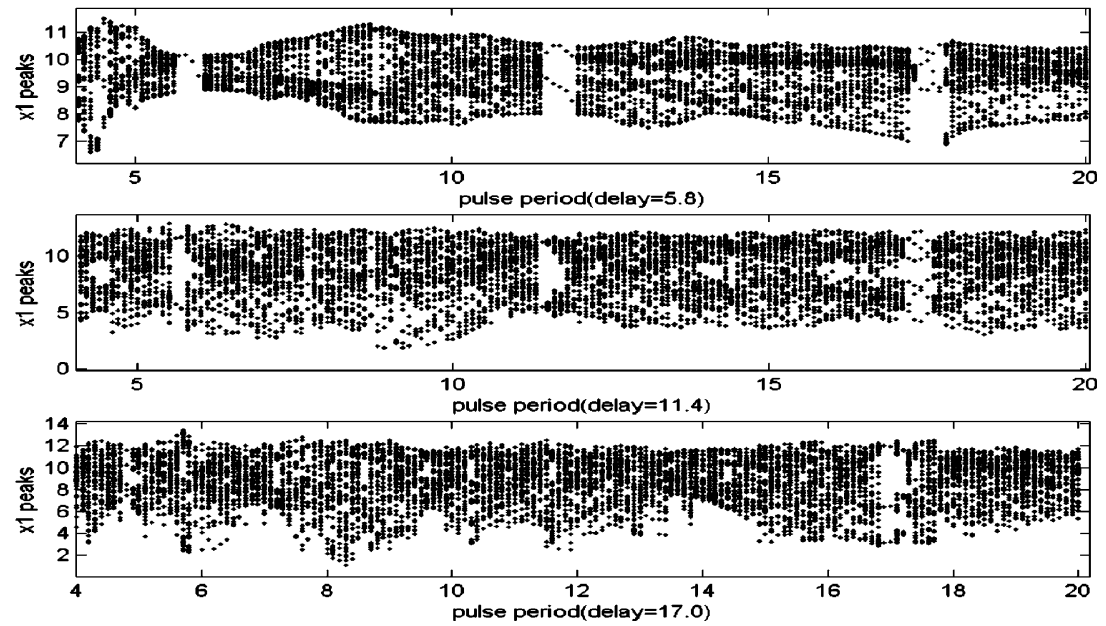

Fig. 14. Dependence of pulse period for different delayed feedback conditions. 

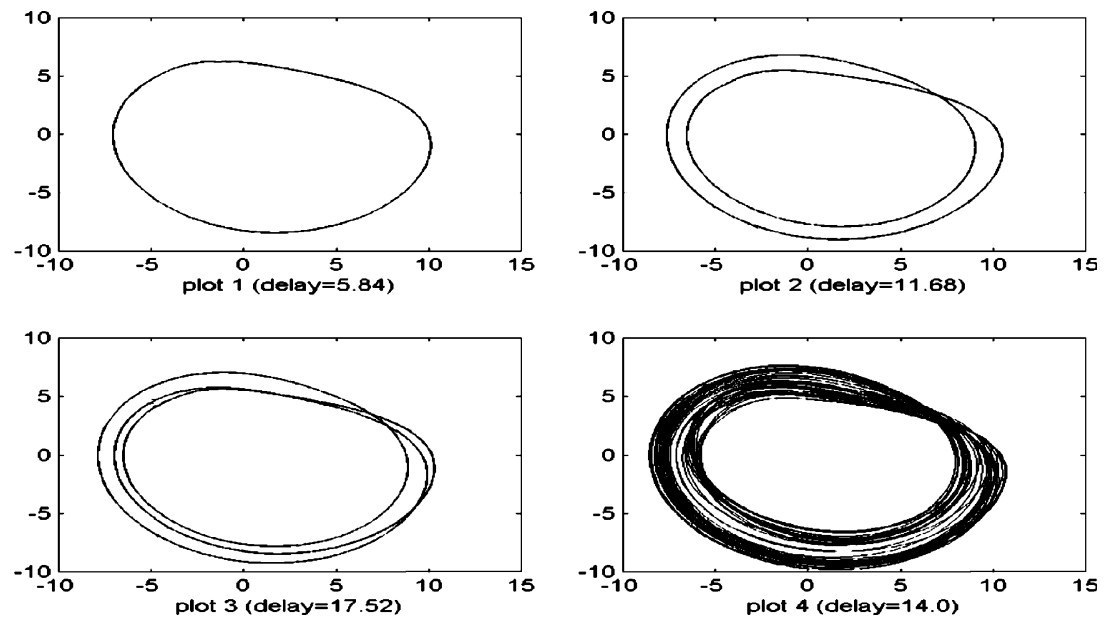

Fig. 15. State space plots for different pulse periods.
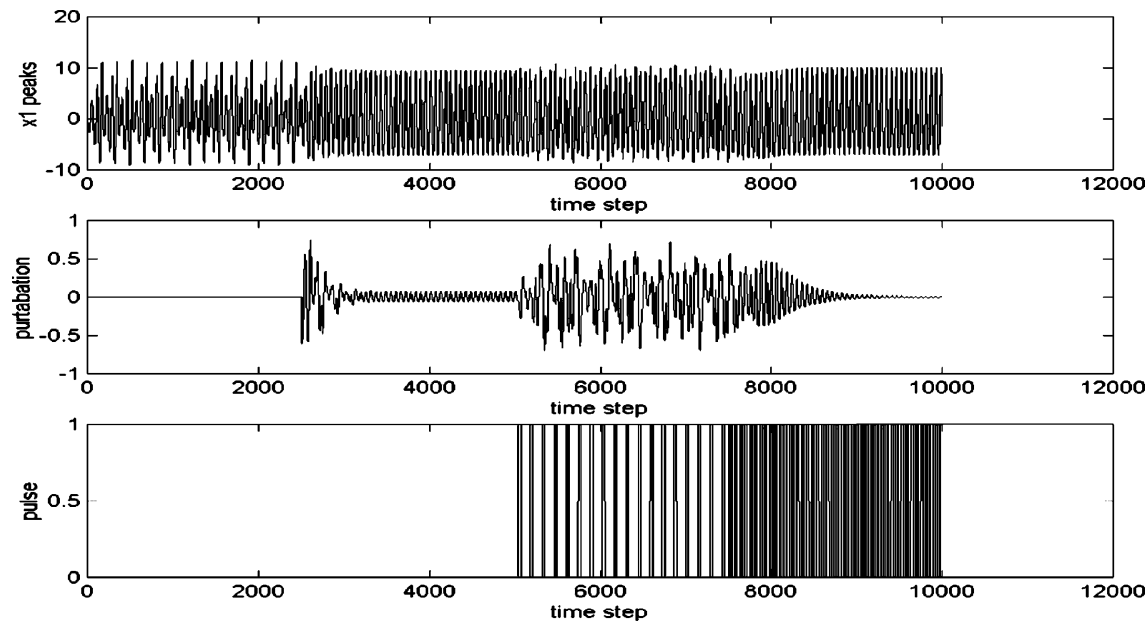

Fig. 16. Time series with perturbation and pulse input.

Feedback control is activated at $t=250$, and the plot of $x_{1}$ shows that the network is stabilised to a period 1 orbit. Note that the once the orbit has been stabilised, the perturbation $F(t)$ quickly becomes small and periodic. The external input is activated at $t=500$ with a pulse period of 14 . At this point the perturbation becomes large and non-periodic again as it unsuccessfully attempts to stabilise an orbit. At $t=750$, the pulse period of the external input is 

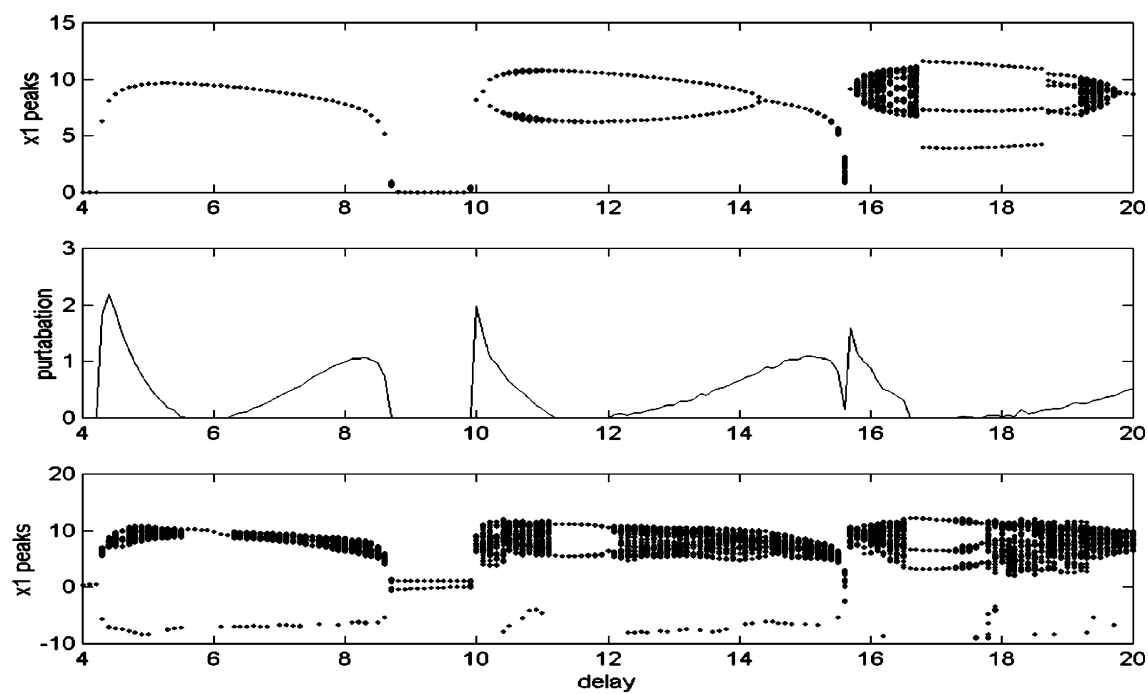

Fig. 17. Relationship between external input and perturbation.

changed to 5.84, which is equal to the feedback delay. The period one orbit then returns and the perturbation $F(t)$ is quickly minimised.

In a final set of experiments we explored the relationship between the delayed feedback control (perturbation) and the external input. In this case the feedback delay $\tau$ and the pulse period of the external input $I(t)$ were given the same value for each experiment. As with previous experiments, the transients were allowed to decay before the feedback control and the external input were synchronously activated. We plotted peak $x_{1}$ and perturbation values for each experiment. The experiments were repeated for $\tau=0$ through to $\tau=20$ with increments of 0.1 . The top plot of Fig. 17 shows the bifurcation diagram when there is no external input and delayed feedback control is activated with $k=0.2$. This plot demonstrates that it is possible to stabilise orbits for a wide range of delays. The middle plot shows the average magnitude of the perturbation for these experiments. The bottom plot shows the bifurcation diagram when the delayed feedback and external input are both on. It is clear that when periodic external input is added to a chaotic system under delayed feedback control it is only possible to stabilise orbits when the average perturbation is at a minimum.

\section{Conclusion}

We have presented a first step towards developing a chaotic neural network which is able to select internal dynamic states in response to external input in a 
self-organised manner. Our results indicate that for the Rössler system it is possible to devise a method of chaos control which self-selects values for $\tau$ corresponding to the nearest UPO which can be stabilised with relatively small fluctuations in the feedback signal $F(t, \tau)$. We have also demonstrated that a network can self-select a UPO in response to specific input values represented by frequency modulations in an external input signal.

The models we have presented here are small and limited. Yet we believe that the underlying principle that UPOs represent memory states and that adaptive feedback delay periods can be used to stabilise them is a very promising area of research in the context of chaotic neural networks. We are currently experimenting with other models which use adaptive feedback delay periods to select UPOs. These models focus on the adaptation of both $\tau_{n}$ and $k_{n}$ parameters in networks with $n$ delays.

\section{References}

[1] N.T. Crook, C.H. Dobbyn, T. olde Scheper, Chaos as a desirable stable state of artificial neural networks, in: R. John, R. Birkenhead (Ed.), Advances in Soft Computing: Soft Computing Techniques and Applications, J. Kacprzyk (Series Editor), Physica-Verlag, New York, 2000, pp. 52-60.

[2] W.L. Ditto, S.N. Rauseo, M.L. Spano, Experimental control of chaos, Physics Review Letters 65 (1990) 3211-3214.

[3] W.J. Freeman, Strange attractors in the olfactory system of rabbits, Electroencephalography and Clinical Neurophysiology 61 (1985) S155-S155.

[4] W.J. Freeman, Neural networks and chaos, Journal of Theoretical Biology 171 (1994) 13-18.

[5] A. Garfinkel, M.L. Spano, W.L. Ditto, J.N. Weiss, Controlling cardiac chaos, Science 257 (1992) 1230-1235.

[6] M.R. Guevara, L. Glass, M.C. Mackey, A. Shrier, Chaos in neurobiology, IEEE Transactions on Systems, Man and Cybernetics SMC-13 (1983) 790-798.

[7] D.O. Hebb, The Organization of Behaviour: A Neurophysiological Theory, John Wiley, New York, 1948.

[8] A. Kittel, M. Popp, J. Parisi, K. Pyragas, Control of chaos by self-adapted delayed feedback, Lecture Notes in Physics 976 (1996) 239-247.

[9] C. Lourenco, A. Babloyantz, Control of spatiotemporal chaos in neuronal networks, International Journal of Neural Systems 7 (1996) 507-517.

[10] T. olde Scheper, Application of Chaos analysis on patient EEG, MSc Thesis, University of Utrecht, 1995.

[11] K. Pyragas, Continuous control of chaos by self-controlling feedback, Physics Letters 170A (1992) 421-428.

[12] O.E. Rössler, An equation for continuous chaos, Physics Letters A 57 (1976) 397-398.

[13] T. Shinbrot, W.L Ditto, C. Grebogi, E. Ott, M.L. Spano, J.A. Yorke, Using the sensitive dependence of chaos (the "butterfly effect") to direct trajectories in an experimental chaotic system, Physical Review Letters 68 (1992) 2863-2866.

[14] T. Shinbrot, C. Grebogi, E. Ott, J.A. Yorke, Using small perturbations to control chaos, Nature 363 (1993) 411-417.

[15] I. Tsuda, E. Koerner, H. Shimizu, Memory dynamics in asynchronous neural networks, Progress of Theoretical Physics 78 (1987) 51-71. 
[16] I. Tsuda, Dynamic link of memory—chaotic memory map in nonequilibrium neural networks, Neural Networks 5 (1992) 313-326.

[17] I. Tsuda, A new-type of self-organization associated with chaotic dynamics in neural networks, International Journal of Neural Systems 7 (1996) 451-459. 